\title{
Association between insulin resistance and arterial stiffness in Mexican patients without type 2 diabetes
}

\author{
Roopa Mehta, ${ }^{1,2 *}$ Neftali E. Antonio-Villa, ${ }^{1,3}$ Omar Y. Bello-Chavolla, ${ }^{4}$ Alexandro J. Martagón, ${ }^{1,5}$ \\ Daniel Elias-López, ${ }^{1,2}$ Arsenio Vargas-Vázquez, ${ }^{1,3}$ Donaji V. Gómez-Velasco, ${ }^{1}$ Paloma Almeda-Valdés, ${ }^{1,2}$ \\ Liliana Muñoz-Hernandez, ${ }^{1,2}$ Ivette Cruz-Bautista, ${ }^{1,2}$ and Carlos A. Aguilar-Salinas ${ }^{1,2,5}$ \\ ${ }^{1}$ Metabolic Diseases Research Unit, Instituto Nacional de Ciencias Médicas y Nutrición Salvador Zubirán; ${ }^{2}$ Endocrinology and Metabolism

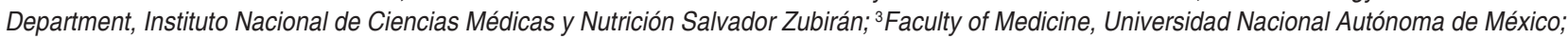 \\ ${ }^{4}$ Research Division, Instituto Nacional de Geriatría; ${ }^{5}$ School of Medicine and Health Sciences Tec Salud, Instituto Tecnológico y de Estudios \\ Superiores de Monterrey. Mexico City, Mexico
}

\begin{abstract}
Background: Central aortic arterial stiffness (CAAS) is an independent cardiovascular risk factor. Insulin resistance (IR) contributes to CAAS-associated risk. Objective: To evaluate the association between IR and CAAS in a Mexican population without diabetes. Methods: IR was estimated with Homeostatic Model Assessment 2-Insulin Resistance (HOMA2-IR) and other surrogate markers (Metabolic score for IR [METS-IR], Quantitative Insulin Sensitivity Check Index [QUICKI], triglycerides/ glucose index [TyG], TyG*body mass index [TyG*BMI] and triglycerides/high-density lipoprotein cholesterol ratio [TG/HDL-C]). CAAS was evaluated using carotid-femoral pulse wave velocity analysis $\left(P W V_{c \mid}\right)$ and the standardized augmentation index (Al-75). Bivariate correlations were made between surrogate markers and PWV ${ }_{c f}$ Increased CAAS was defined as PWV ${ }_{c f}$ above the $90^{\text {th }}$ percentile. Thresholds and area under the curve $(A \cup C)$ were obtained for each surrogate marker in order to evaluate their performance in estimating increased CAAS. Results: Three hundred and fifty-eight patients were included. A correlation was found between HOMA2-IR and PWV ${ }_{o p}$ this correlation was replicated with other surrogate markers. METS-IR and TyG*B$M I$ had the highest degree of correlation with $P W V_{c f}$ When adjustments were made for covariates, the correlations with TyG*BMI, METS-IR, HOMA2-IR and QUICKI maintained significance. HOMA2-IR showed the strongest correlation with Al-75. METS-IR and TyG showed the best AUC. Patients with prediabetes had the highest PWV conclusions: The relationship between IR and CAAS is present before the onset of diabetes; this association may entail higher cardiovascular risk.
\end{abstract}

KEY WORDS: Insulin resistance Arterial stiffness. Prediabetes. Cardiometabolic profile.

\section{La asociación entre la resistencia a la insulina y la rigidez arterial en pacientes mexicanos sin diabetes mellitus tipo 2}

\section{Resumen}

Antecedentes: La rigidez arterial central aórtica (RACA) es un factor de riesgo cardiovascular independiente. La resistencia a la insulina (RI) contribuye al riesgo asociado a RACA. Objetivo: Evaluar la asociación entre Rl y RACA en una población mexicana sin diabetes. Métodos: La RI se estimó con HOMA2-IR y (Homeostatic Model Assessment 2-Insulin Resistance) otros subrogados (METS-IR [Metabolic score for IR], QUICKI [Quantitative Insulin Sensitivity Check Index], TyG [ratio triglicéridos/glucosa], TyG*IMC [TyG*índice de masa corporal] y TG/HDL [ratio TG/lipoproteínas de alta densidad]). Se evaluó la RACA mediante el análisis de velocidad de onda del pulso carotídeo-femoral $\left(V P_{c f}\right.$ ) y el índice de aumentación estandarizado (Al-75).

\footnotetext{
Correspondence:

Date of reception: 17-03-2021

*Roopa Mehta

E-mail: roopamehta@yahoo.com license (http://creativecommons.org/licenses/by-nc-nd/4.0/).
} 
Se realizaron correlaciones bivariante entre los subrogados y la $V O P_{c f}$ RACA aumentada se definió como VOP arriba del percentil 90. Se obtuvieron puntos de corte y área bajo la curva $(A B C)$ para cada subrogado para estimar RACA aumentada. Resultados: Se incluyó 358 pacientes. Se encontró una correlación entre HOMA2-IR y VOP ${ }_{c \beta}$ esta correlación se replicó con Ios subrogados. METS-IR y TyG*IMC tuvieron el mayor grado de correlación con VOP ${ }_{\text {cf }}$ Al ajustar, las correlaciones con TyG*IMC, METS-IR, HOMA2-IR y QUICKI mantuvieron significancia. La correlación con Al-75 fue mayor para HOMA2-IR. METSIR y TyG mostraron la mejor ABC. Los pacientes con prediabetes tuvieron mayor VOP ${ }_{\text {cf }}$ Conclusiones: La relación entre la Rl y la RACA está presente desde etapas no diabéticas; esta asociación puede conllevar mayor riesgo cardiovascular.

PALABRAS CLAVE: Resistencia a la insulina. Rigidez arterial. Prediabetes. Perfil cardiometabólico.

\section{Introduction}

Insulin resistance (IR) is characterized by a decrease in tissue sensitivity to insulin. It is associated with type 2 diabetes (T2D), obesity and metabolic syndrome; this parameter contributes to the cardiovascular (CV) risk associated with these conditions..$^{1-3}$ The pathophysiological mechanisms involved in the relationship between IR and CV morbidity and mortality are not well known. ${ }^{4}$ Some researchers have explored the association between IR and central aortic arterial stiffness (CAAS). ${ }^{5}$

The arterial system maintains adequate blood flow and has a "cushioning or dampening" function. ${ }^{6}$ This depends on arterial elasticity. Arteries can lose their vascular compliance with age and with hypertension, chronic kidney disease, T2D and atherosclerosis. ${ }^{7}$ CAAS is an independent CV risk factor. ${ }^{8,9}$ When an artery is rigid, forward pulse wave is faster and is reflected from the periphery more rapidly and reaches the heart during early systole, thus producing an increase in systolic blood pressure; at the same time, diastolic pressure is reduced and there is a decrease in coronary diastolic perfusion, ${ }^{10}$ in addition to the transmission of more pulsatile energy to the small arteries, which causes microvascular damage.

The gold standard for measuring insulin sensitivity is euglycemic-hyperinsulinemic clamp. ${ }^{11}$ This method is not useful in clinical practice because it is laborious, invasive and high-priced. ${ }^{12} \mathrm{~A}$ widely used surrogate index for estimating IR is the Homeostatic Model Assessment for Insulin Resistance (HOMA2-IR). Other indices such as the Quantitative Insulin Check Index (QUICKI), and surrogate markers that use metabolic and anthropometric parameters in their formulas (e.g., Metabolic score for IR [METS-IR], triglycerides/glucose ratio [TyG], TyG*body mass index [TyG*BMI] and triglycerides/ high-density lipoprotein cholesterol ratio [TG/HDL-C] $)^{13-15}$ also have a good correlation with the clamp method.
Carotid-femoral pulse wave velocity $\left(\mathrm{PWV}_{\mathrm{cf}}\right)$ is the gold standard for CAAS non-invasive evaluation..$^{16,17}$ The role played by IR in promoting CAAS increase has not been clarified. In addition, this relationship may be biased by the sum of metabolic alterations seen in patients with T2D. The purpose of this study is to evaluate the association between IR and CAAS $\left(P W V_{c f}\right)$ in a Mexican population without diabetes.

\section{Methodology}

\section{Design and study population}

A cross-sectional study was conducted with subjects being recruited between January 2017 and December 2020 at the Metabolic Diseases Research Unit of Salvador Zubirán National Institute of Medical Sciences and Nutrition. Participants were aged between 18 and 70 years and had no T2D previous diagnosis. Patients with previous prediabetes diagnoses (fasting glucose levels of $100-125 \mathrm{mg} / \mathrm{dL}$ or glycated hemoglobin [HbA1c] of 5.7-6.4\%), hypertension (blood pressure $\geq 140 / 80 \mathrm{mmHg}$ and/or on treatment with antihypertensive drugs), obesity (BMl $\geq 30 \mathrm{~kg} / \mathrm{m}^{2}$ ), and subjects with high $\mathrm{CV}$ risk (e.g., atherogenic primary dyslipidemia) were included. Individuals with CV disease, chronic kidney disease and life expectancy of less than one year were excluded. The study was carried out in accordance with the statutes of the Declaration of Helsinki. All participants signed an informed consent document prior to participating in the study. Salvador Zubirán National Institute of Medical Sciences Ethics Committee approved the study.

\section{Biochemical, anthropometric evaluation}

Venous blood samples were obtained after an 8 to 12 hour fasting period. Plasma glucose analysis was carried out using an automated analyzer (Yellow Springs Instruments, Yellow Springs, $\mathrm{OH}, \mathrm{USA}$ ), 
insulin concentrations were measured by chemiluminescence immunoassay (Beckman Coulter Access 2), $\mathrm{HbA1C}$ levels were measured by chromatography (Variant II Turbo, BIORAD) and lipid profile concentrations were measured by colorimetry assays (Unicel DxC 600 Synchron Clinical System Beckman Coulter). Low-density lipoprotein cholesterol (LDL-C) was calculated according to Martín's formula. ${ }^{18}$ All subjects were weighted using SECA mBCA 514 calibrated scales and measured with SECA stadiometers. Waist circumference was measured using a non-elastic tape between the intersecting midpoint of the ribcase margin and the upper edge of the rib. BMI was calculated by dividing weight in kilograms by height in squared meters. The HOMA2-IR index was used as the standard to evaluate IR. Subsequently, the association was replicated using the QUICKI, TG/HDL-C, TyG, TyG*BMI and METS-IR surrogate markers. Table 1 shows the formulas for IR surrogate markers estimation.

\section{Carotid wave analysis evaluation}

Subjects were asked not to consume caffeine and to refrain from smoking for the previous 48 hours. Carotid wave analysis was carried out using a semiautomatic device (SphygmoCore XCEL, AtCor Medical Pty Ltd, USA). At the time of evaluation of the participants, they were placed in the supine position for 10 minutes. A sphygmomanometer was placed between the proximal third of the patient's right leg to record the femoral wave. $\mathrm{PWV}_{\mathrm{cf}}$ and the standardized augmentation index at 75 beats per minute (Al-75) (to reduce the effect attributable to heart rate) were measured by flattening tonometry using the carotid-to-femoral arterial wave corrected time of delay. ${ }^{19}$ The augmentation index estimates the reflection of the peripheral wave; earlier return occurs with increased pulse velocity. Arterial stiffness was defined as those subjects with $\mathrm{PWV}_{\text {cf }}$ above the $90^{\text {th }}$ percentile $(p \geq 90)(>7.77 \mathrm{~mm} / \mathrm{s})$.

\section{Statistical analysis}

Qualitative variables were expressed as absolute count and percentage; quantitative variables, as the mean (standard deviation) or median (interquartile range $[I Q R])$ according to their normal distribution. Log transformations were applied. Subjects with arterial stiffness were compared with those without arterial stiffness using Student's t or Mann-Whitney's U statistical tests.
Table 1. Formulas of surrogated indices for estimating insulin resistance. Glucose, TG and HDL-C units are expressed in $\mathrm{mg} / \mathrm{dL}$. Insulin units expressed in IU/mL

\begin{tabular}{|c|c|}
\hline Index & Formula \\
\hline HOMA2-IR & https://www.dtu.ox.ac.uk/homacalculator/ \\
\hline \multirow[t]{2}{*}{ METS-IR } & $\left(\operatorname{Ln}\left[\left(2^{\star}\right.\right.\right.$ glucose $)+$ triglycerides $\left.]\right){ }^{\star} B M I$ \\
\hline & $\overline{L n(H D L \text { cholesterol })}$ \\
\hline \multirow[t]{2}{*}{ QUICKI } & 1 \\
\hline & $\overline{(\log (\text { insulin })+\log (\text { glucose }))}$ \\
\hline TyG & $\operatorname{Ln}\left(\right.$ triglycerides * $\left.\frac{[\text { glucose }]}{2}\right)$ \\
\hline TyG*BMI & $(T y G)^{*}(B M I)$ \\
\hline TG/HDL-C & (Triglycerides) / (HDL cholesterol) \\
\hline \multicolumn{2}{|c|}{$\begin{array}{l}\text { HOMA2-IR: Homeostatic Model Assessment 2-Insulin Resistance; METS-IR: Metabolic } \\
\text { score for IR; QUICKI: Quantitative Insulin Sensitivity Check Index; TyG: triglycerides/ } \\
\text { glucose ratio; TyG*BMI: TyG*body mass index; TG/HDL-C: TG/high-density lipoprotein } \\
\text { cholesterol ratio. }\end{array}$} \\
\hline
\end{tabular}

\section{Correlation between insulin resistance surrogate markers}

The correlation of HOMA2-IR and the other surrogate markers with $\mathrm{PWV}_{\mathrm{cf}}$ and AI-75 was evaluated. Correlations were adjusted for age, gender, hypertension, smoking, HbA1c, waist circumference and dyslipidemia. To evaluate the differences between subjects with IR with our surrogate markers, the cutoff points previously published by Almeda-Valdez et al. ${ }^{20}$ were used. PWV $_{\text {cf }}$ parameters were compared between subjects with IR using Student's t-test.

\section{Evaluation of cutoff points for identifying arterial stiffness}

Cutoff points for identifying arterial stiffness were calculated. Sensitivity, specificity, positive and negative predictive value of each IR surrogate marker were estimated. The area under the curve (AUC) of each surrogate marker was obtained. Statistical analysis was carried out with the $\mathrm{R}$ programming language (Version 3.6.1).

\section{Results}

Three hundred and fifty-eight subjects were included, out of whom 260 (72.6\%) were women, with median 
Table 2. General characteristics of the study population

\begin{tabular}{|c|c|c|c|c|}
\hline Parameter & $\begin{array}{l}\text { Total population } \\
\qquad(\mathrm{n}=358)\end{array}$ & $\begin{array}{l}\text { No arterial stiffness } \\
\qquad(n=323)\end{array}$ & $\begin{array}{l}\text { Arterial stiffness } \\
\quad(n=35)\end{array}$ & p-value \\
\hline Female gender, $\mathrm{n}(\%)$ & $260(72.6 \%)$ & $238(75.1)$ & $18(51.4)$ & 0.001 \\
\hline Age, years (range) & $50(39-57)$ & $46.6(12.7)$ & $53(7.65)$ & $<0.001$ \\
\hline Prediabetes, n (\%) & $254(70.9)$ & $223(70.3)$ & $26(74.3)$ & 0.627 \\
\hline Weight, kg (SD) & $76.2( \pm 14.9)$ & $75.7( \pm 14.9)$ & $81.7( \pm 15.8)$ & 0.005 \\
\hline Waist, cm (SD) & $95.5( \pm 12.1)$ & $93.6( \pm 11.7)$ & $98.8( \pm 11.3)$ & 0.003 \\
\hline BMl, kg/m² (range) & $28.9(26.4-32.2)$ & $28(26-32)$ & $29.2(26.6-35)$ & 0.065 \\
\hline Glucose, mg/dL (SD) & $96.3( \pm 12.8)$ & $95.6( \pm 11.1)$ & $102.2( \pm 22.6)$ & 0.004 \\
\hline Triglycerides, mg/dL (range) & $137(95-187)$ & $133(92-182)$ & $159(108-197)$ & 0.093 \\
\hline Total cholesterol, mg/dL (SD) & $197( \pm 43.1)$ & $194( \pm 42)$ & $188( \pm 45.8)$ & 0.239 \\
\hline HDL cholesterol, mg/dL (SD) & $46.3( \pm 12.2)$ & $46.7( \pm 12)$ & $41.9( \pm 12)$ & 0.027 \\
\hline LDL cholesterol, mg/dL (SD) & $123( \pm 35.5)$ & $121( \pm 35)$ & $116( \pm 35.7)$ & 0.320 \\
\hline Insulin, IU/dL (range) & $8.9(6-12.9)$ & $8.7(5.7-11.9)$ & $9.9(6.8-14.9)$ & 0.313 \\
\hline $\mathrm{HbA1c}, \%(\mathrm{SD})$ & $5.8( \pm 0.75)$ & $5.8( \pm 0.60)$ & $6.3( \pm 1.18)$ & $<0.001$ \\
\hline Uric acid, mg/dL (SD) & $5.4( \pm 1.3)$ & $5.3( \pm 1.29)$ & $5.6( \pm 1.36)$ & 0.343 \\
\hline Creatinine, mg/dL (SD) & $0.87( \pm 1.9)$ & $0.86( \pm 2.45)$ & $0.81( \pm 0.21)$ & 0.712 \\
\hline AST, mg/dL (SD) & $28.2( \pm 16.2)$ & $28.3( \pm 17)$ & $27.8( \pm 8.2)$ & 0.879 \\
\hline ALT, mg/dL (SD) & $28.3( \pm 19.9)$ & $28.3( \pm 17)$ & $30.1( \pm 15.9)$ & 0.534 \\
\hline GGT, mg/dL (SD) & $26.4( \pm 26.2)$ & $25.9( \pm 26.8)$ & $28.9( \pm 21.7)$ & 0.550 \\
\hline APO B, mg/dL (SD) & $112.25( \pm 28.4)$ & $109( \pm 27.7)$ & $102( \pm 29.5)$ & 0.321 \\
\hline $\mathrm{PWV}_{\mathrm{cf}}, \mathrm{mm} / \mathrm{s}(\mathrm{SD})$ & $6.52( \pm 1.34)$ & $5.92( \pm 0.84)$ & $8.58( \pm 0.65)$ & $<0.001$ \\
\hline $\mathrm{Al}-75, \%$ (SD) & $34.97( \pm 13.22)$ & $34.3( \pm 13.9)$ & $33.7( \pm 9.71)$ & 0.604 \\
\hline HOMA2-IR (range) & $1.2(0.8-1.7)$ & $1.2(0.8-1.6)$ & $1.3(0.9-2.0)$ & 0.144 \\
\hline METS-IR (SD) & $45.6( \pm 9.47)$ & $45.1( \pm 9.25)$ & $48.8( \pm 10.5)$ & 0.026 \\
\hline QUICKI (SD) & $0.344( \pm 0.033)$ & $0.346( \pm 0.033)$ & $0.335( \pm 0.033)$ & 0.079 \\
\hline TyG (SD) & $8.78( \pm 0.58)$ & $8.73( \pm 0.58)$ & $8.99( \pm 0.57)$ & 0.075 \\
\hline TyG*BMI (SD) & $259.8( \pm 49.4)$ & $257.4( \pm 47.63)$ & $273.9( \pm 54.4)$ & 0.057 \\
\hline TG/HDL-C (range) & $2.99(1.8-4.6)$ & $2.8(1.7-4.5)$ & $3.8(2.4-5.8)$ & 0.017 \\
\hline
\end{tabular}

age being 50 years (IQR: 39-57). Population characteristics are shown in table 2. The subjects with greater arterial stiffness were older men, with higher weight, waist circumference and with a higher concentration of fasting glucose, $\mathrm{TG}$ and $\mathrm{HbA1c}$, and lower concentrations of HDL-C. PWV ${ }_{\text {cf }}$ and Al-75 mean values in this population were $6.52( \pm 1.34) \mathrm{mm} / \mathrm{s}$ and 34.9
$( \pm 13.22) \%$, respectively. Regarding the surrogate indices (Table 1), mean/median values were: HOMA2-IR, 1.2 (IQR: 0.8-1.7); METS-IR, 45.6 ( \pm 9.47); QUICKI, 0.344 ( \pm 0.03); TyG, $8.78( \pm 0.58) ;$ TyG*BMI, $259.8( \pm$ 49.4); and TG/HDL-C, 2.99 (IQR: 1.8-4.6). Only the METS-IR and TG/HDL-C indices were significantly related to greater arterial stiffness. 

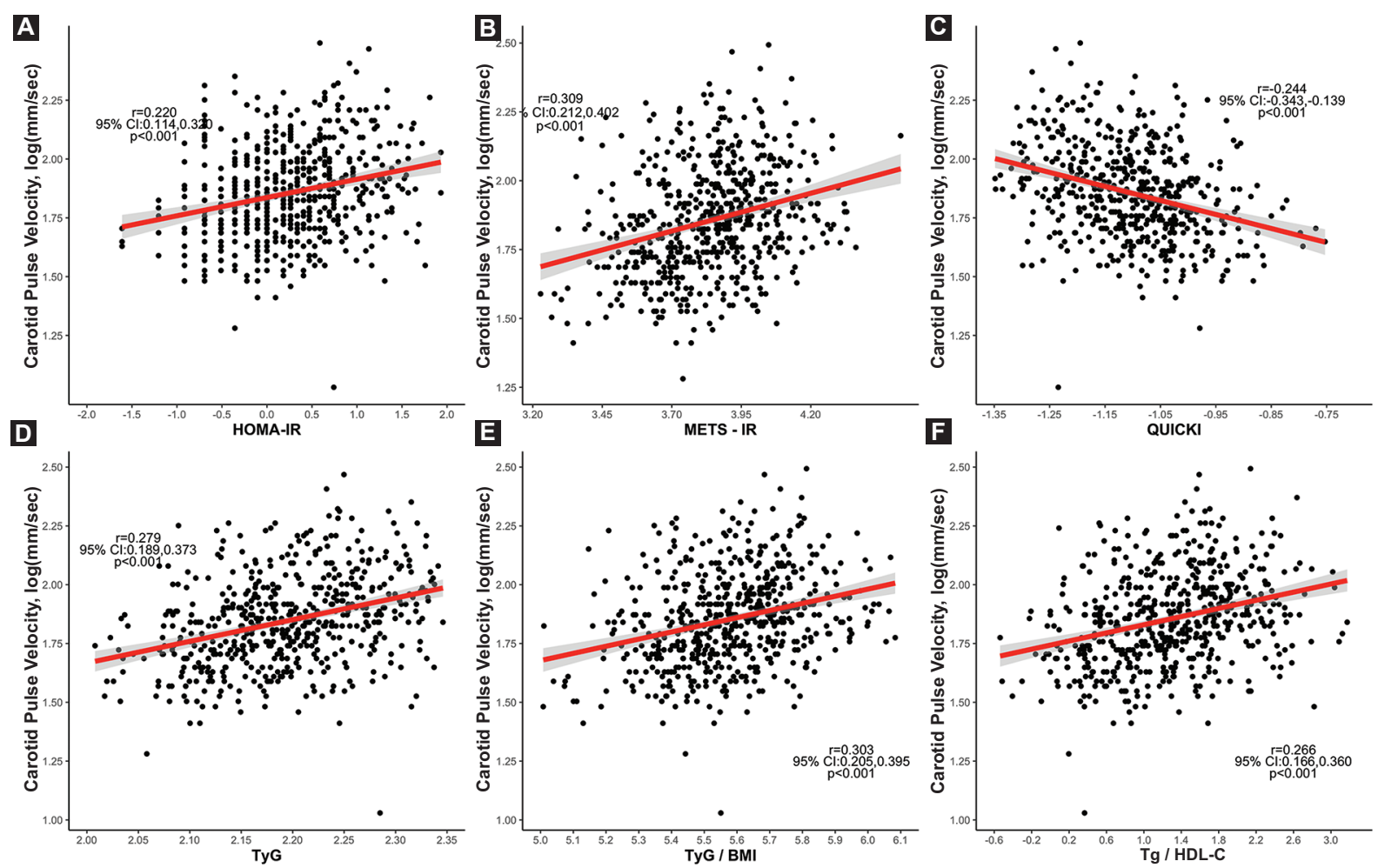

Figure 1. Insulin resistance surrogate markers correlation with carotid pulse velocity. The variables were transformed to their log values to reach parametric distribution. Cl: confidence interval; HOMA2-IR: Homeostatic Model Assessment 2-Insulin Resistance; METS-IR: Metabolic score for IR; QUICKI: Quantitative Insulin Sensitivity Check Index; TyG: triglycerides/glucose ratio; TyG*BMI: TyG*body mass index; $T g / H D L-C$ : $T g$ / high-density lipoprotein cholesterol ratio.

\section{Carotid pulse velocity correlation with insulin resistance surrogate markers}

In the bivariate correlation analysis, a correlation was observed with HOMA2-IR and $\mathrm{PWV}_{\text {cf }}$ (r: 0.220; 95\% confidence interval [Cl]: 0.114-0.320), which was maintained when adjustments were made for covariates (r: 0.128; $95 \% \mathrm{Cl}: 0.018-0.234)$. The association was replicated with the rest of the surrogate indices. The highest correlation was observed for METS-IR index (r: 0.309; 95\% Cl: 0.212-0.402) and TyG*BMI index (r: 0.279; 95\% Cl: 0.189-0.373) (Fig. 1). For Al75 , the TyG*BMI index had the highest degree of correlation (r: 0.131; 95\% Cl: 0.027-0.231). However, when adjustments were made for covariates, the HOMA2-IR index maintained the strongest association with Al-75 (r: 0.168; 95\% Cl: 0.060-0.272) (Table 3). Finally, patients with IR had an increase in $\mathrm{PWV}_{\text {cf }}$ values evaluated by all surrogate markers (Fig. 2). When $\mathrm{PWV}_{\text {cf }}$ values were explored in subjects with prediabetes, we confirmed that this population had greater arterial stiffness in comparison with those without prediabetes (Fig. 3).

\section{Cutoff points for identifying arterial stiffness}

The best cutoff point for detecting arterial stiffness was determined for each surrogate marker. The HOMA2-IR index showed adequate predictive performance. This association was replicated for the rest of IR surrogate markers. The indices with the best area under the curve (AUC) were the METS-IR index (AUC: $0.61 ; 95 \% \mathrm{Cl}: 0.51-0.71$ ) and TyG index (AUC: $0.61 ; 95 \% \mathrm{Cl}: 0.51-0.69)$. The surrogate index with the highest sensitivity, with a significant AUC, was the TyG index (S: $0.94 ; 95 \% \mathrm{Cl}: 0.81-0.99$ ), while the TyG*BMl index had the best specificity (Sp: 0.84; 95\% Cl: 079-0.88) with a significant AUC (Table 4).

\section{Discussion}

The association between IR and pulse wave velocity was evaluated in a Mexican population without T2D. Patients with IR, evaluated by surrogate markers, had an increase in $P W V_{\text {of }}$ values that was independent of 
Table 3. Pearson's correlation between insulin resistance surrogate markers and arterial stiffness estimate. Adjustment variables are: age, gender, active smoking, hypertension, glycated hemoglobin and waist circumference

\begin{tabular}{|c|c|c|c|c|}
\hline \multirow[t]{2}{*}{ Surrogate markers } & \multicolumn{2}{|c|}{$\mathrm{PWV}_{\text {of }}$} & \multicolumn{2}{|c|}{ Al-75 } \\
\hline & Bivariate & Adjusted & Bivariate & Adjusted \\
\hline HOMA2-IR & $\begin{array}{c}0.220 \\
(0.114,0.320)\end{array}$ & $\begin{array}{c}0.128 \\
(0.018,0.234)\end{array}$ & $\begin{array}{c}0.033 \\
(-0.074,0.141)\end{array}$ & $\begin{array}{c}0.168 \\
(0.060,0.272)\end{array}$ \\
\hline METS-IR & $\begin{array}{c}0.309 \\
(0.212,0.402)\end{array}$ & $\begin{array}{c}0.171 \\
(0.063,0.275)\end{array}$ & $\begin{array}{c}0.099 \\
(0.018,0.178)\end{array}$ & $\begin{array}{c}0.164 \\
(0.082-0.241)\end{array}$ \\
\hline QUICKI & $\begin{array}{c}-0.244 \\
(-0.343,-0.139)\end{array}$ & $\begin{array}{c}-0.119 \\
(-0.226,-0.01)\end{array}$ & $\begin{array}{c}-0.063 \\
(-0.145,0.019)\end{array}$ & $\begin{array}{c}-0.144 \\
(-0.225,-0.062)\end{array}$ \\
\hline TyG & $\begin{array}{c}0.279 \\
(0.189,0.373)\end{array}$ & $\begin{array}{c}0.081 \\
(-0.027,0.189)\end{array}$ & $\begin{array}{c}0.021 \\
(-0.083,0.124)\end{array}$ & $\begin{array}{c}0.126 \\
(0.018,0.232)\end{array}$ \\
\hline TyG*BMl & $\begin{array}{c}0.303 \\
(0.205,0.395)\end{array}$ & $\begin{array}{c}0.183 \\
(0.053,0.286)\end{array}$ & $\begin{array}{c}0.131 \\
(0.027,0.231)\end{array}$ & $\begin{array}{c}0.164 \\
(0.059,0.269)\end{array}$ \\
\hline TG/HDL-C & $\begin{array}{c}0.266 \\
(0.166,0.360)\end{array}$ & $\begin{array}{c}0.085 \\
(-0.024,0.193)\end{array}$ & $\begin{array}{c}-0.042 \\
(-0.145,0.062)\end{array}$ & $\begin{array}{c}0.118 \\
(0.009,0.223)\end{array}$ \\
\hline
\end{tabular}

$\mathrm{PWV}_{\mathrm{cf}}$ : carotid-femoral pulse wave velocity; AI-75: standardized augmentation index; HOMA2-IR: Homeostatic Model Assessment 2-Insulin Resistance; METS-IR: Metabolic score for IR; QUICKI: Quantitative Insulin Sensitivity Check Index; TyG: triglycerides/glucose ratio; TyG*BMI: TyG*body mass index; TG/HDL-C: TG/high-density lipoprotein cholesterol ratio.
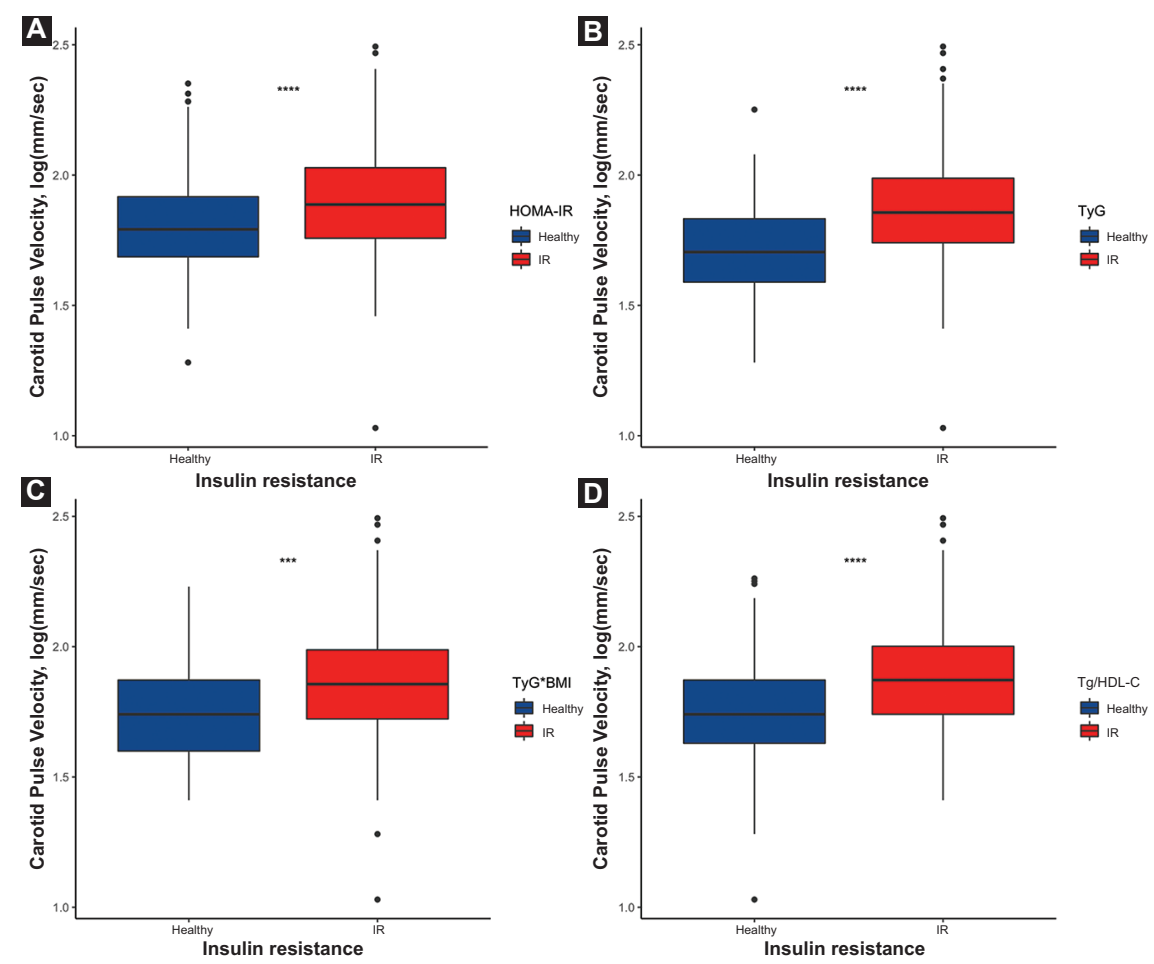

Figure 2. Carotid pulse velocity differences between subjects with insulin resistance evaluated by surrogate marker.

${ }^{*} p<0.05$.

${ }^{t} p<0.001$.

$\neq p<0.0001$.

IR: insulin resistance; HOMA2-IR: Homeostatic Model Assessment 2-Insulin Resistance; TyG: triglycerides/glucose ratio; TyG*BMI: TyG*body mass index; Tg/HDL-C: TG/high-density lipoprotein cholesterol ratio.

risk factors. The indices with the highest degree of association were METS-IR and TyG*BMI, and those with the best AUC for detecting arterial stiffness were
METS-IR and TyG. Subjects with prediabetes had greater arterial stiffness in comparison with those without prediabetes. 

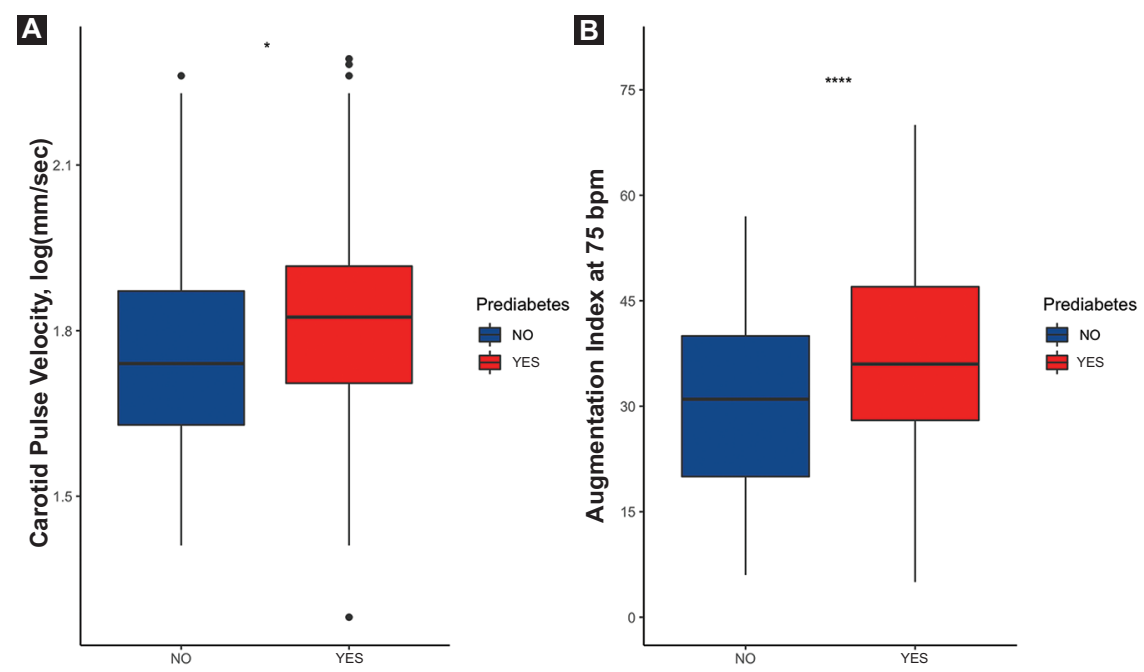

Figure 3. Differences in carotid pulse velocity (A) and augmentation index at 75 bpm (B) between subjects with and without prediabetes. ${ }^{*} p<0.05$.

$+p<0.001$

$\neq p<0.0001$.

Table 4. Cutoff points for identifying arterial stiffness $\left(\mathrm{PWV}_{\mathrm{cf}} \mathrm{p} \geq 90\right)$ in healthy subjects. Arterial stiffness was defined as those subjects with PWVcf above the $90^{\text {th }}$ percentile $(p>90)$ with a value of 7.77

\begin{tabular}{|c|c|c|c|c|c|c|}
\hline Variables & Cutoff point & Sensitivity (\%) & Specificity (\%) & PPV (\%) & NPV (\%) & AUC \\
\hline HOMA2-IR & 1.7 & $\begin{array}{c}0.43 \\
(0.26-0.62)\end{array}$ & $0.76(0.71-0.81)$ & $\begin{array}{c}0.17 \\
(0.14-0.30)\end{array}$ & $\begin{array}{c}0.92 \\
(0.85-0.94)\end{array}$ & $\begin{array}{c}0.59 \\
(0.48-0.69)\end{array}$ \\
\hline METS-IR & 41.6 & $\begin{array}{c}0.82 \\
(0.66-0.93)\end{array}$ & $\begin{array}{c}0.39 \\
(0.33-0.48)\end{array}$ & $\begin{array}{c}0.13 \\
(0.11-0.31)\end{array}$ & $\begin{array}{c}0.95 \\
(0.89-0.96)\end{array}$ & $\begin{array}{c}0.61 \\
(0.51-0.71)\end{array}$ \\
\hline QUICKI & 0.39 & $\begin{array}{c}0.13 \\
(0.04-0.29)\end{array}$ & $\begin{array}{c}0.91 \\
(0.88-0.94)\end{array}$ & $\begin{array}{c}0.14 \\
(0.10-0.32)\end{array}$ & $\begin{array}{c}0.91 \\
(0.71-0.94)\end{array}$ & $\begin{array}{c}0.40 \\
(0.29-0.51)\end{array}$ \\
\hline TyG & 8.4 & $\begin{array}{c}0.94 \\
(0.81-0.99)\end{array}$ & $\begin{array}{c}0.27 \\
(0.22-0.32)\end{array}$ & $\begin{array}{c}0.27 \\
(0.10-0.55)\end{array}$ & $\begin{array}{c}0.98 \\
(0.92-0.98)\end{array}$ & $\begin{array}{c}0.61 \\
(0.51-0.70)\end{array}$ \\
\hline TyG*BMI & 301.9 & $\begin{array}{c}0.34 \\
(0.19-0.52)\end{array}$ & $\begin{array}{c}0.84 \\
(0.79-0.88)\end{array}$ & $\begin{array}{c}0.19 \\
(0.15-0.33)\end{array}$ & $\begin{array}{c}0.92 \\
(0.84-0.94)\end{array}$ & $\begin{array}{c}0.59 \\
(0.48-0.69)\end{array}$ \\
\hline TG/HDL-C & 3.05 & $\begin{array}{c}0.69 \\
(0.51-0.83)\end{array}$ & $\begin{array}{c}0.52 \\
(0.47-0.58)\end{array}$ & $\begin{array}{c}0.14 \\
(0.11-0.27)\end{array}$ & $\begin{array}{c}0.94 \\
(0.88-0.95)\end{array}$ & $\begin{array}{c}0.60 \\
(0.51-0.70)\end{array}$ \\
\hline
\end{tabular}

PWV : carotid-femoral pulse wave velocity; PPV: positive predictive value; NPV: negative predictive value; AUC: area under the curve; HOMA2-IR: Homeostatic Model Assessment 2-Insulin Resistance; METS-IR: Metabolic score for IR; QUICKI: Quantitative Insulin Sensitivity Check Index; TyG: triglycerides/glucose ratio; TyG*BMI: TyG*body mass index; TG/HDL-C: TG/high-density lipoprotein cholesterol ratio.

A direct relationship between $\mathrm{PWV}_{\mathrm{cf}}$ and atherosclerosis has been shown and, in longitudinal studies, this parameter is an independent $\mathrm{CV}$ risk marker. ${ }^{21} \mathrm{An}$ increase in arterial stiffness may be one of the mechanisms that explains the association between $\mathrm{CV}$ risk and IR. Epidemiological studies have shown that IR is an independent factor for the development of arterial stiffness. In the ARIC trial, individuals with T2D or carbohydrate intolerance had stiffer arteries than subjects with normoglycemia. ${ }^{22}$ It was speculated that the effect of glucose, insulin and TG together play a role in the development of arterial stiffness. Significant differences have been shown in $\mathrm{PWV}_{\text {of }}$ between subjects with and without metabolic syndrome. ${ }^{23}$ The specific mechanisms involved are not clear, but we do know that IR has direct and indirect effects on vasculature. Hyperinsulinemia can increase sympathetic tone, activate the renin-angiotensin system, stimulate vascular inflammation and reduce flow-mediated endothelium-dependent vasodilation. The end result is endothelial dysfunction and an inadequate vasomotor response to a 
pro-inflammatory and pro-coagulant endothelium, which increases the risk for arterial stiffness. ${ }^{24}$

Several authors have evaluated the association between IR surrogate markers and arterial stiffness. In subjects without CV disease, a significant association between TyG and PWV ${ }_{c f}$ has been confirmed. ${ }^{25} \mathrm{HO}$ MA2-IR was independently associated with $\mathrm{PWV}_{c f}$ in individuals without diabetes or with prediabetes. ${ }^{26,27}$ Webb et al. evaluated PWV $_{\text {cf }}$ in subjects with normoglycemia, abnormal glucose regulation (abnormal fasting glucose and/or carbohydrate intolerance) and TD2. ${ }^{28}$ In the latter two groups, there was a significant association with arterial stiffness. Glucose intolerance and HOMA2-IR were the most important independent predictors of arterial stiffness.

This study has some limitations. Surrogate markers were used for $\mathrm{RI}$ in replacement of the gold standard. The population was heterogeneous and may not be representative of the general population. The majority were women, which represents a potential selection bias. Data on exercise and medications that could influence the atherosclerosis process and IR were not obtained.

\section{Conclusions}

This is the first study in a Mexican population that confirms the relationship between IR and arterial stiffness in people without diabetes. The fact that the relationship between IR and arterial stiffness is present before the onset of diabetes is corroborated; this association may entail higher $\mathrm{CV}$ risk in the future. The HOMA2-IR index showed an association that was replicated with the different IR surrogate markers. The best performance for detecting arterial stiffness was with METS-IR and TyG indices. These are easy-to-calculate parameters and can be included in CV risk assessment.

\section{Acknowledgements}

Neftali Eduardo Antonio-Villa and Arsenio Vargas-Vázquez belong to the MD/PhD PECEM program of UNAM Faculty of Medicine. Neftali Eduardo Antonio-Villa and Arsenio Vargas-Vázquez are supported by CONACyT.

\section{Funding}

This research has not received any specific grant from agencies of the public, commercial or non-profit sectors.

\section{Conflict of interests}

The authors declare that they have no conflicts of interest.

\section{Ethical disclosures}

Protection of human and animal subjects. The authors declare that no experiments were performed on humans or animals for this research.

Confidentiality of data. The authors declare that they have followed the protocols of their work center on the publication of patient data.

Right to privacy and informed consent. The authors have obtained informed consent from the patients and/or subjects referred to in the article. This document is in the possession of the corresponding author.

\section{References}

1. Howard G, O'Leary DH, Zaccaro D, Haffner S, Rewers M, Hamman R, et al. Insulin sensitivity and atherosclerosis. Circulation. 1996;93:1809-17.

2. Adeva-Andany MM, Martínez-Rodríguez J, González-Lucán $M$, Fernández-Fernández C, Castro-Quintela E. Insulin resistance is a cardiovascular risk factor in humans. Diabetes Metab Syndr. 2019;13(2):1449-55.

3. Poon AK, Meyer ML, Tanaka H, Selvin E, Pankow J, Zeng D, et al. Association of insulin resistance, from mid-life to late-life, with aortic stiffness in late-life: the Atherosclerosis Risk in Communities Study. Cardiovasc Diabetol. 2020;19:11.

4. Fu S, Lin Y, Luo L, Ye P. Relationship between central arterial stiffness and insulin resistance in Chinese community-dwelling population without diabetes mellitus. Int J Endocrinol. 2017;2017:1073919.

5. Cruickshank K, Riste L, Anderson SG, Wright JS, Dunn G, Gosling RG. Aortic pulse-wave velocity and its relationship to mortality in diabetes and glucose intolerance: an integrated index of vascular function? Circulation. 2002;106(16):2085-90

6. Gajdova J, Karasek D, Goldmannova D, Krystynik O, Schovanek J, Vaverkova $\mathrm{H}$, et al. Pulse wave analysis and diabetes mellitus. A systematic review. Biomed Pap Med Fac Univ Palacky Olomouc Czech Repub. 2017;161(3):223-33.

7. Janić M, Lunder M, Sabovič M. Arterial stiffness and cardiovascular therapy. Biomed Res Int. 2014;2014:621437.

8. Vlachopoulos C, Aznaouridis K, Stefanadis C. Prediction of cardiovascular events and all-cause mortality with arterial stiffness: a systematic review and meta-analysis. J Am Coll Cardiol. 2010;55:1318-27.

9. Ben-Shlomo Y, Spears M, Boustred C, May M, Anderson SG, Benjamin EJ, et al. Aortic pulse wave velocity improves cardiovascular event prediction: an individual participant meta-analysis of prospective observational data from 17,635 subjects. J Am Coll Cardiol. 2014:63:636-46.

10. Faconti L, Nanino E, Mills CE, Cruickshank KJ. Do arterial stiffness and wave reflection underlie cardiovascular risk in ethnic minorities? JRSM Cardiovasc Dis. 2016;5:1-9.

11. Defronzo R, Tobin J, Andres R. Glucose clamp technique: a method for quantifying insulin secretion and resistance. Am $J$ Physiol.1979;237:E214-23.

12. Otten J, Ahrén B, Olsson T. Surrogate measures of insulin sensitivity vs the hyperinsulinaemic-euglycaemic clamp: a meta-analysis. Diabetologia. 2014;57(9):1781-8.

13. Fiorentino TV, Marini MA, Succurro E, Andreozzi F, Sest G. Relationships of surrogate indexes of insulin resistance with insulin sensitivity assessed by euglycemic hyperinsulinemic clamp and subclinical vascular damage. BMJ Open Diabetes Res Care. 2019;7(1):e000911.

14. Borai A, Livingstone C, Kaddam I, Ferns G. Selection of the appropriate method for the assessment of insulin resistance. BMC Med Res Methodol. 2011;11:158-67.

15. Bello-Chavolla OY, Almeda-Valdés P, Gómez-Velasco D, Viveros-Ruiz T, Cruz-Bautista I, Romo-Romo A, et al. METS-IR, a novel score to evaluate insulin sensitivity, is predictive of visceral adiposity and incident type 2 diabetes. Eur J Endocrinol. 2018;178(5):533-44. 
16. Townsend RR, Wilkinson IB, Schiffrin EL, Avolio AP, Chirinos JA, Cockcroft JR, et al. Recommendations for improving and standardizing vascular research on arterial stiffness: a scientific statement from the American Heart Association. Hypertension. 2015:66:698-722.

17. Townsend RR. Arterial stiffness: Recommendations and standardization. Pulse. 2016;4(suppl 1):3-7.

18. Martin SS, Blaha MJ, Elshazly MB, Toth PP, Kwiterovich PO, Blumenthal RS, et al. Comparison of a novel method vs the Friedewald equation for estimating low-density lipoprotein cholesterol levels from the standard lipid profile. J Am Med Assoc. 2013;310:2061-8.

19. Van Bortela LM, Laurent S, Boutouyrie P, Chowienczyk P, Cruickshank JK De Backera T, et al.; on behalf of the Artery Society, the European Society of Hypertension Working Group on Vascular Structure and Function and the European Network for Noninvasive Investigation of Large Arteries. Expert consensus document on the measurement of aortic stiffness in daily practice using carotid-femoral pulse wave velocity. J Hypertension. 2012;30:445-8.

20. Almeda-Valdés $P$, Bello-Chavolla OY, Caballeros-Barragán CR, Gómez-Velasco DV, Viveros-Ruiz T, Vargas-Vázquez A, et al. Índices para la evaluación de la resistencia a la insulina en individuos mexicanos sin diabetes. Gac Med Mex. 2018;154(Supp 2):S50-S55.

21. Kim HL, Kim SH. Pulse wave velocity in atherosclerosis. Front Cardiovasc Med. 2019;6:41
22. Salomaa V, Riley W, Kark JD, Nardo C, Folsom AR. Non-insulin-dependent diabetes mellitus and fasting glucose and insulin concentrations are associated with arterial stiffness indexes. The ARIC Study. Atherosclerosis Risk in Communities Study. Circulation. 1995;91(5):1432-43.

23. Peñaherrera $C A$, Peñaherrera $R$, Duarte $M C$, Peñaherrera $E$. Assessment of arterial stiffness in patients with metabolic syndrome in Ecuador: A cross-sectional study. Diabetes Metab Syndr. 2017;11(3):199-202.

24. Novo G, Manno G, Russo R, Buccheri D, Dell'Oglio S, Morreale P, et al. Impact of insulin resistance on cardiac and vascular function. Int J Cardiol. 2016;221:1095-9.

25. Won KB, Park GM, Lee SE, Cho IJ, Kim HC, Lee BK, et al. Relationship of insulin resistance estimated by triglyceride glucose index to arterial stiffness. Lipids Health Dis. 2018;17(1):268.

26. Fu S, Lin Y, Luo L, Ye P. Relationship between Central Arterial Stiffness and Insulin Resistance in Chinese Community-Dwelling Population without Diabetes Mellitus. Int J Endocrinol. 2017;2017:1073919.

27. Ho CT, Lin CC, Hsu HS, Liu CS, Davidson LE, Li TC, et al. Arterial stiffness is strongly associated with insulin resistance in Chinese - A population-based study (Taichung Community Health Study, TCHS). J Atheroscler Thromb. 2011;18 (2):122-30.

28. Webb DR, Khunti K, Silverman R, Gray LJ, Srinivasan B, Lacy PS, et al. Impact of metabolic indices on central artery stiffness: independent association of insulin resistance and glucose with aortic pulse wave velocity. Diabetologia. 2010;53:1190-8. 